\title{
Infant Botulism in the Very Young Neonate: A Case Series
}

\author{
Laura Jackson, $\mathrm{MD}^{1}$ Suneeta Madan-Khetarpal, $\mathrm{MD}^{2}$ \\ Marian G. Michaels, MD, $\mathrm{MPH}^{4}$ Melissa Riley, MD ${ }^{1}$ \\ ${ }^{1}$ Division of Newborn Medicine, Department of Pediatrics, \\ Children's Hospital of Pittsburgh of UPMC, Pittsburgh, Pennsylvania \\ 2 Division of Medical Genetics, Department of Pediatrics, Children's \\ Hospital of Pittsburgh of UPMC, Pittsburgh, Pennsylvania \\ ${ }^{3}$ Division of Neurology, Department of Pediatrics, Children's Hospital \\ of Pittsburgh of UPMC, Pittsburgh, Pennsylvania \\ ${ }^{4}$ Division of Infectious Disease, Department of Pediatrics, Children's \\ Hospital of Pittsburgh of UPMC, Pittsburgh, Pennsylvania \\ Am J Perinatol Rep 2017;7:e163-e166.
}

Monica Naik, MD³

Address for correspondence Laura Jackson, MD, Division of Newborn Medicine, Department of Pediatrics, Children's Hospital of Pittsburgh of UPMC, 4401 Penn Avenue, Pittsburgh, PA 15224

(e-mail: laura.jackson@chp.edu).

\begin{abstract}
Keywords

- neonatology

- infant botulism

- BIG-IV

Background Though botulism is a rare disease overall, all infants younger than 1 year of age are at risk of contracting infant botulism, the most prevalent form reported in the United States. Nonetheless, infant botulism is frequently omitted from the differential diagnosis of the very young neonate exclusively due to age, and the diagnosis is often only considered secondarily after a costly and prolonged work up is undertaken. Delayed diagnosis can lead not only to unnecessary testing but also to prolonged hospital stay and increased morbidity.

Case This case series highlights three critically ill neonates, all younger than 30 days, who presented to our neonatal intensive care unit and were eventually diagnosed with infant botulism. The initial diagnostic dilemma is described for each of these patients and highlights the importance of early recognition of the main symptoms, in addition to consideration of important potential coinciding conditions.
\end{abstract}

Botulism is a rare but potentially lethal disease that can affect all ages. Of the four forms of botulism (infant, foodborne, wound, and intestinal), the infant form is the most common in the United States. ${ }^{1,2}$ The average age at presentation is 16 weeks, with the range being younger than 1 week up to 60 weeks, ${ }^{3,4}$ and incubation can be as short as 3 days. ${ }^{3}$ There are few sporadic cases reported in infants younger than 30 days. ${ }^{4,5}$ The classic presentation of symmetric descending paralysis is often initially absent in young infants with the disease, and is frequently replaced by a poorly defined chief complaint, including poor feeding and lethargy. The rarity of infant botulism in this population, coupled with the vague symptomatology and lack of obvious spore exposure, often makes botulism a forgotten possibility on the differential diagnosis of the presenting neonate. Three cases of infant botulism presenting for medical evaluation between 19 to 26 days of life are presented.

\section{Case 1}

A 26-day-old term male patient presented with a 1-week history of congestion and cough, and a 1-day history of decreased intake, activity, and urine output. There was no fever, and multiple family members had upper respiratory infections. The infant was switched to a soy-based formula 1 week prior for concerns of colic and inconsistent bowel movements. Upon admission, he was sleepy but arousable with normal vital signs and a mildly prolonged capillary refill. He had a rounded abdomen with decreased bowel sounds. Neurological examination revealed diffusely decreased tone with weak deep tendon reflexes. Initial diagnostic evaluation is listed in - Table 1. The infant was started on ampicillin and cefepime. After 1 hour of admission, the infant had apnea requiring endotracheal intubation and received

April 24, 2017

accepted after revision

June 14, 2017
DOI https://doi.org/

10.1055/s-0037-1604407. ISSN 2157-6998.
Copyright $\odot 2017$ by Thieme Medical Publishers, Inc., 333 Seventh Avenue, New York, NY 10001, USA. Tel: +1(212) 584-4662.
License terms

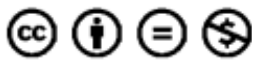


Table 1 Initial diagnostic work up obtained in each patient

\begin{tabular}{|c|c|c|c|}
\hline & Patient 1 & Patient 2 & Patient 3 \\
\hline Blood culture & $\mathrm{N}$ & $\mathrm{N}$ & $\mathrm{N}$ \\
\hline CSF culture & $\mathrm{N}$ & $\mathrm{N}$ & $\mathrm{N}$ \\
\hline Urine culture & $\mathrm{N}$ & $\mathrm{N}$ & $\mathrm{N}$ \\
\hline $\mathrm{CBC}$ & $\mathrm{N}$ & $\mathrm{N}$ & $\mathrm{N}$ \\
\hline Blood gas (initial) & $\mathrm{N}$ & $\mathrm{N}$ & $\mathrm{N}$ \\
\hline Electrolytes & $\mathrm{N}$ & $\mathrm{N}$ & $\mathrm{N}$ \\
\hline Respiratory viral swab & $\mathrm{N}$ & $P$ & - \\
\hline CSF cell count & $\mathrm{N}$ & - & $\mathrm{N}$ \\
\hline Liver function tests & $\mathrm{N}$ & $\mathrm{N}$ & $\mathrm{N}$ \\
\hline Lactate & $\mathrm{N}$ & - & $\mathrm{N}$ \\
\hline Pyruvate & - & - & $\mathrm{N}$ \\
\hline Ammonia & $\mathrm{N}$ & - & $\mathrm{N}$ \\
\hline CPK & $\mathrm{N}$ & $\mathrm{N}$ & $\mathrm{N}$ \\
\hline Acylcarnitine profile & $\mathrm{N}$ & - & $\mathrm{N}$ \\
\hline Amino acid profile & $\mathrm{N}$ & - & $\mathrm{N}$ \\
\hline Urine organic acids & $\mathrm{N}$ & - & $\mathrm{N}$ \\
\hline Microarray & $\mathrm{N}$ & - & $\mathrm{N}$ \\
\hline Chest X-ray & N & $\begin{array}{l}\text { Left retrocardiac opacity } \\
\text { concerning for pneumonia }\end{array}$ & $\begin{array}{l}\text { Right diaphragm slightly } \\
\text { higher than left }\end{array}$ \\
\hline Abdominal X-ray & $\begin{array}{l}\text { Moderate amount of formed } \\
\text { stool throughout colon }\end{array}$ & - & - \\
\hline EEG & $\mathrm{N}$ & $\mathrm{N}$ & - \\
\hline Head ultrasound & - & $\mathrm{N}$ & - \\
\hline Brain MRI & $\mathrm{N}$ & - & $\mathrm{N}$ \\
\hline
\end{tabular}

Abbreviations: -, not performed; CBC, complete blood count; CPK, creatinine phosphokinase; CSF, cerebral spinal fluid; EEG, electroencephalogram; $\mathrm{MRI}$, magnetic resonance imaging; $\mathrm{N}$, normal/negative; $\mathrm{P}$, positive.

mechanical ventilation. The infant's neurological examination continued to deteriorate; deep tendon reflexes became diffusely absent, pupils were sluggishly responsive to light, and the infant no longer had a gag reflex or significant spontaneous movement. On the seventh day of admission, infant botulism was considered and stool was positive for botulinum toxin type $\mathrm{B}$, confirming the diagnosis. Due to the continued need for mechanical ventilation and lack of significant clinical improvement, botulism immune globulin intravenous (BIG-IV) was administered. The infant was able to be extubated on hospitalization day 27, and was discharged 36 days after presentation.

\section{Case 2}

A 21-day-old term male patient was admitted to the intensive care unit following an apneic event. Parents noted the infant developed drooling, poor feeding, and less vigorous extremity movement of a few days duration. He had also reportedly stopped crying 1 week prior and had not stooled for 3 days. The social history was unremarkable except that the father was a pipelayer. Initial examination revealed an ill-appearing infant with hypotonia and shallow breathing, but stable vital signs.
Initial work up is outlined in - Table 1. Ampicillin and gentamicin were initiated. On the second day of admission, apneic episodes led to endotracheal intubation and mechanical ventilation. A left retrocardiac opacity on chest radiograph indicated pneumonia, and the respiratory viral panel confirmed rhinovirus. The infant was extubated on hospitalization day 11 , but required support via noninvasive positive pressure ventilation. He continued to have generalized hypotonia with poor head control and a weak gag reflex. An electromyogram (EMG) was obtained and showed short, brief motor unit potentials with some polyphasia in all muscles tested. As this finding was suspicious for botulism, a stool sample was sent and was positive for botulinum toxin type B on hospitalization day 19. BIG-IV was not administered, as the patient had already started to show improvement with supportive care. He was discharged to home 47 days after admission.

\section{Case 3}

A 19-day-old term male patient presented to the hospital for polysomnography per parental request due to a family history of sudden infant death syndrome (SIDS). Upon presentation, the infant was mottled with diffuse hypotonia, 
and was transferred to the emergency department for evaluation. He had little-to-no movement of the lower extremities, and decreased tone with absent deep tendon reflexes; however, his facial movements were symmetric with full extraocular movements. Soon after arrival, the infant had an apneic event requiring intubation. Family history was notable for a female sibling death from SIDS at 38 days of life. Parents had not appreciated decreased activity or tone in the neonate before presentation; they reported normal stooling and no issues with breastfeeding. Initial work up is listed in - Table 1. Vancomycin and cefotaxime were initiated. A chest radiograph after intubation demonstrated the right diaphragm to be higher than the left; subsequent fluoroscopy revealed minimal movement of the right diaphragm. EMG indicated a generalized myopathic process with axonal sensory-motor polyneuropathy. Muscle biopsy was obtained and showed a nonspecific pattern of myofiber atrophy, but was mostly inconclusive for diagnostic purposes. Cerebral spinal fluid was sent for amino acids, folate, lactate, pyruvate, and neurotransmitters; all studies were within normal limits. Testing for spinomuscular atrophy with respiratory distress (SMARD1) was carried out due to unilateral diaphragmatic involvement, continued peripheral weakness, and lack of pupillary abnormalities or ptosis. The infant's clinical condition remained static, and a stool sample was also sent on admission day 36 , which revealed botulinum toxin type B. BIG-IV was administered due to lack of clinical improvement. Initially, the patient appeared to have improving spontaneous movements, return of some deep tendon reflexes, and spontaneous respirations but continued to remain weak. Approximately 2 weeks after Baby-BIG treatment, SMARD1 was also confirmed as an additional diagnosis on gene testing, with two variants in the IGHMBP2 gene (Cys496X with a premature stop codon in exon 10, pathogenic variant, and Arg425Cys, likely pathogenic in exon 9) in trans, explaining the additional symptoms and need for ongoing support. A gastric feeding tube and tracheostomy were placed. The infant continued to require mechanical ventilation at discharge, 94 days after admission.

\section{Discussion}

Infant botulism is caused by the production of potent botulinum toxin by the bacteria Clostridium botulinum in the gut after ingesting spores. ${ }^{2}$ The neurotoxin is transported through the bloodstream to the presynaptic terminal of the motor neuron, where it irreversibly binds and blocks acetylcholine release. The pathogenesis of infant botulism is unique to this age group, as intestinal colonization with C. botulinum does not occur in adults with a normal intestinal microbiome. ${ }^{6}$

Classically, symptoms of botulism include descending, symmetric paralysis beginning with cranial nerve palsies manifested as ptosis, slow pupillary reflex, and loss of gag reflex, ${ }^{7}$ and eventually leading to loss of deep tendon reflexes and respiratory failure over the course of hours to days. Diaphragmatic weakness is a late manifestation but is typically bilateral in nature if present. Lack of a fever is common.
Constipation, though a well-known symptom of botulism in older children, is often difficult to determine in a neonate with a variable stooling pattern. Exposure to $C$. botulinum spores (e.g., ingestion of honey or exposure to a construction site) is often not identified in confirmed cases, ${ }^{8}$ and the lack of such exposure should not deter the clinician from considering the diagnosis.

Infant botulism is regularly omitted from the differential diagnosis of the very young neonate given the vague presentation. The differential diagnosis includes not only sepsis, but also congenital myopathies, metabolic disorders, and drug exposure. ${ }^{9}$ Our third patient demonstrated some symptoms inconsistent with botulism, including unilateral diaphragmatic involvement and distal digit weakness. Particularly, spinal muscular atrophy (SMA) type 1 is a common mimic of botulism. ${ }^{9}$ Therefore, it is prudent that the clinician be sure that botulism explains all of the neurological findings, and additional diagnoses should be considered even if a botulism diagnosis was confirmed.

While the diagnosis of infant botulism is made based on clinical suspicion, it can be confirmed by identifying botulinum toxin in patient stool or serum, or by isolating botulinum toxin-producing clostridia in patient stool specimens. EMG findings consistent with infant botulism are not pathognomonic and may not be present early in the course of the disease. The typical pattern shows decreased compound muscle action potentials and incremental response to repetitive nerve stimulation. ${ }^{10}$ Though EMG is not necessary to solidify the diagnosis, it can be helpful for timely diagnosis. BIG-IV (human) is obtained from the California Department of Public Health. BIG-IV works to neutralize free botulinum toxin; while it does not immediately improve symptoms, it further prevents the toxin from binding, resulting in faster clinical improvement. Infants that receive BIG-IV have an average hospital stay of 2.6 weeks, whereas infants not given the medication will incur an average hospitalization of 5.7 weeks. $^{11}$ Though administration should not be delayed for confirmation of diagnosis, given the high cost of BIG-IV, clinicians should consult with a specialist before administration. Special care should be taken in selection of antibiotics for patients with this potential diagnosis, as particular classes of medications, such as aminoglycosides, can cause additional toxin release or further prevent acetylcholine release. ${ }^{3}$

While awaiting resolution of symptoms, clinicians should provide appropriate supportive care and monitor for signs of superimposed bacterial infection. Full recovery is expected in infants that do not suffer complications associated with paralysis, such as aspiration or asphyxia. Without supportive care, death is possible, as infant botulism is also known to be a cause of SIDS. ${ }^{2}$

In summary, though infant botulism presents at an average age of 4 months, it should be considered as a diagnosis in neonates, regardless of age, who demonstrate characteristic symptoms and do not recover along the typical timeline for sepsis. ${ }^{4}$ If suspicion for infant botulism is high, BIG-IV should be administered as quickly as possible, even before laboratory confirmation of the disease. Other confirmed diagnoses 
(viral infections, genetic abnormalities, etc.) should not dissuade the clinician from testing for botulism or considering treatment if symptomatology is concerning.

\section{Conflict of Interest}

The authors have no conflicts of interest to disclose.

\section{References}

1 Carrillo-Marquez MA. Botulism. Pediatr Rev 2016;37(05):183-192

2 Arnon S. Infant botulism. In: Cherry JD, ed. Feigin and Cherry's Textbook of Pediatric Infectious Diseases. 7th ed. Philadelphia, PA: Elsevier Saunders; 2013:1801-1809

3 Arnon S, Barzilay E. Clostridial infections: botulism and infant botulism (Clostridium botulinum). In: Kimberlin D, ed. Red Book. 30th ed. Elk Grove Village, IL: American Academy of Pediatrics; 2015:294-296
4 Thilo EH, Townsend SF, Deacon J. Infant botulism at 1 week of age: report of two cases. Pediatrics 1993;92(01):151-153

5 Keet CA, Fox CK, Margeta M, et al. Infant botulism, type F, presenting at 54 hours of life. Pediatr Neurol 2005;32(03): 193-196

6 Burr DH, Sugiyama H. Susceptibility to enteric botulinum colonization of antibiotic-treated adult mice. Infect Immun 1982;36 (01):103-106

7 Fox CK, Keet CA, Strober JB. Recent advances in infant botulism. Pediatr Neurol 2005;32(03):149-154

8 Rosow LK, Strober JB. Infant botulism: review and clinical update. Pediatr Neurol 2015;52(05):487-492

9 Francisco AM, Arnon SS. Clinical mimics of infant botulism. Pediatrics 2007;119(04):826-828

10 Gutierrez AR, Bodensteiner J, Gutmann L. Electrodiagnosis of infantile botulism. J Child Neurol 1994;9(04):362-365

11 Arnon SS, Schechter R, Maslanka SE, Jewell NP, Hatheway CL. Human botulism immune globulin for the treatment of infant botulism. N Engl J Med 2006;354(05):462-471 